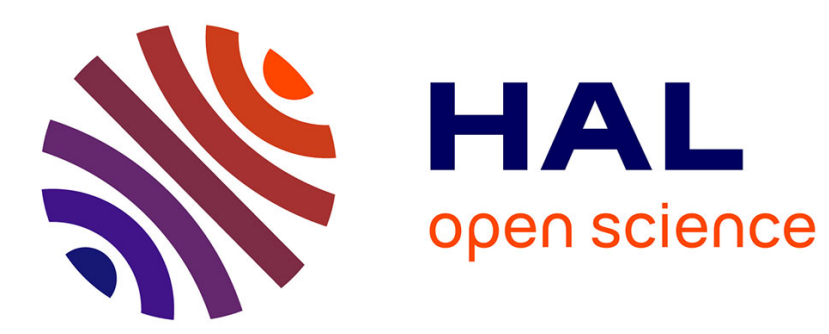

\title{
Mesurer les préférences du consommateur pour orienter les décisions des pouvoirs publics: l'apport de la méthode expérimentale
}

\author{
Stéphane Robin, Anne Rozan, Bernard Ruffieux
}

\section{To cite this version:}

Stéphane Robin, Anne Rozan, Bernard Ruffieux. Mesurer les préférences du consommateur pour orienter les décisions des pouvoirs publics : l'apport de la méthode expérimentale. 2007. hal-00196310

\author{
HAL Id: hal-00196310 \\ https://hal.science/hal-00196310
}

Submitted on 12 Dec 2007

HAL is a multi-disciplinary open access archive for the deposit and dissemination of scientific research documents, whether they are published or not. The documents may come from teaching and research institutions in France or abroad, or from public or private research centers.
L'archive ouverte pluridisciplinaire HAL, est destinée au dépôt et à la diffusion de documents scientifiques de niveau recherche, publiés ou non, émanant des établissements d'enseignement et de recherche français ou étrangers, des laboratoires publics ou privés. 
Mesurer les préférences du consommateur pour orienter les décisions des pouvoirs publics : l'apport de la méthode expérimentale

Stéphane Robin, Anne Rozan, Bernard Ruffieux

Juillet 2007

GATE Groupe d'Analyse et de Théorie Économique UMR 5824 du CNRS

93 chemin des Mouilles - 69130 Écully - France

B.P. 167 - 69131 Écully Cedex

Tél. +33 (0)4 72866060 - Fax +33 (0)4 72866090

Messagerie électronique gate@gate.cnrs.fr

Serveur Web : www.gate.cnrs.fr 


\title{
Mesurer les préférences du consommateur pour orienter les décisions des pouvoirs publics : l'apport de la méthode expérimentale ${ }^{1}$
}

\section{Using experimental method to elicit consumer preferences for public decision}

\author{
Stéphane Robin (*), Anne Rozan (**), Bernard Ruffieux (***)
}

Juillet 2007

\section{Résumé :}

La prise en compte de l'intérêt du citoyen dans la décision publique passe par l'estimation des préférences individuelles face à différents choix collectifs. Dès lors qu'il n'existe pas de marché de référence, mesurer l'impact d'une décision publique sur le bien-être des individus pose un vrai problème méthodologique. En dépit des nombreuses améliorations apportées à ces méthodes, l'enquête d'opinion ou l'évaluation contingente produisent des mesures altérées par différents biais. L'économie expérimentale constitue une méthodologie intéressante en permettant une mesure directe de la valeur qu'attribue un individu pour un choix, un scénario ou un bien non marchand. L'utilisation de cette méthode comme outil d'aide à la décision publique est croissante. Nous nous proposons dans cet article de faire une présentation de cette démarche. Précisément, cet article répond aux deux questions suivantes : Pourquoi faire de l'évaluation des préférences en laboratoire ? Comment conduire une telle investigation ?

Mots clefs : Décision publique, Economie expérimentale, Evaluation de préférences individuelles

* GATE, Université de Lyon, CNRS, ENS-LSH, Centre Léon Bérard

** GSP UMR ENGEES-CEMAGREF, Strasbourg

*** GAEL, UMR INRA et Grenoble Universités

\footnotetext{
${ }^{1}$ Nous tenons à remercier l'éditeur de la revue ainsi que deux rapporteurs anonymes pour leurs commentaires et suggestions.
} 


\begin{abstract}
:
Individual preferences must be evaluated to take into account the citizens' interests for public decisions. Usually, economists assess individual preferences through market decisions. But more often, public decisions impact goods without market prices. Without a market to refer to, evaluating the consequences of a public decision on individual welfare is a challenging methodological problem. In spite of major improvements, preference studies built on hypothetical questions (survey or contingent hypothetical valuations) are biased. Experimental economics is a promising alternative to obtain directly individual valuation for a choice, a scenario or a nonmarket good. Preference study in the lab based on the experimental method is a growing field. This paper aims to present this approach. Precisely, this paper answers the following questions: Why is it interesting to use the experimental method to elicit individual preferences? And how to conduct this category of experiments?
\end{abstract}

Key words: Public Decision, Experimental economics, Preference Elicitation

Code JEL : B4, C9, D1 
Pourquoi, du point de vue des pouvoirs publics, s’intéresser aux préférences des consommateurs ? Les préférences des consommateurs ne sont-elles pas, en principe, une question de nature privée, relevant de la seule sphère marchande ? En fait, les pouvoirs publics ont au moins deux raisons légitimes de s'intéresser aux préférences individuelles.

Tout d’abord, les marchés sont encadrés par une législation importante et évolutive. Lorsqu'il envisage, par exemple, la mise en place d'une nouvelle règle d'étiquetage ou une nouvelle norme de qualité, le décideur public doit pouvoir anticiper l’impact de sa réforme sur les préférences et les comportements des consommateurs. Ensuite, une part importante du champ d'action des pouvoirs publics porte sur la mise à disposition de biens publics. Dans une logique d'optimisation des arbitrages, il s'agit de mettre en balance le coût de cette mise à disposition et les avantages pour les utilisateurs. Là encore, le décideur doit pouvoir disposer d’une information fiable sur les préférences des individus.

S’il est légitime d'étudier les préférences individuelles des consommateurs pour orienter certains choix publics en matière de régulation des marchés ou de mise à disposition de biens publics, comment procéder pour connaître ces préférences ? Une manière possible est de mesurer ces préférences via les consentements à payer individuels (CAP). Le CAP se définit comme le prix maximum que nous serions prêts à payer, dans des conditions normales de marché, pour acquérir un bien ou un service, une caractéristique spécifique d’un produit ou encore une information. Toute une série de raisons rendent ces CAP difficiles d'accès pour un observateur extérieur. D’abord, chacun d'entre nous n'est pas habitué à se poser de façon systématique la question de ses CAP : chacun sait en général que le prix du pain est bien inférieur à son CAP et que le prix d'un week-end ordinaire aux Seychelles est bien supérieur à son CAP. Ces montants ne sont donc pas facilement mobilisables sur le plan cognitif. Il nous faut exercer un effort d'introspection important pour savoir réellement combien nous sommes prêts à payer au maximum pour obtenir un bien ou un service donné. Ensuite, il existe des raisons objectives et stratégiques pour qu'un individu n’ait pas intérêt à révéler ouvertement son CAP. Dans certaines situations, il aura intérêt à le minimiser s’il anticipe que cette valeur pourra engendrer dans l'avenir une baisse de son bien être, comme la création d'une nouvelle taxe. Dans d'autres situations, il exagérera son CAP, s’il anticipe que cela conduira à la mise à disposition d'un nouveau bien public dont il n’aura pas à payer directement le coût associé.

De nombreuses méthodologies ont été mises au point et largement utilisées pour les pouvoirs publics pour mesurer des CAP. Questionnaires, enquêtes, évaluations contingentes ont été utilisés avec plus ou moins de bonheur. Le présent article fait le point sur ces 
différentes méthodes et sur leurs limites. Une autre méthode, plus récente, possède des qualités que les méthodes antérieures n'avaient pas. C’est l'objet principal de cet article que de présenter cette méthode et d'en montrer les propriétés, les usages mais aussi ses limites. Cette méthode, issue de l'économie expérimentale, consiste à créer le marché qui n'existe pas. Ce marché est créé de toutes pièces, pour quelques heures, pour quelques dizaines de consommateurs, dans un laboratoire contrôlé par le chercheur. Cette création n’a qu’une seule finalité : observer de véritables comportements d'achat sur ces marchés afin de pouvoir utiliser la propriété unique des marchés qu'est sa capacité à révéler les CAP individuels. L’organisation de ces marchés en laboratoire permet de contrôler au mieux les variables décisionnelles qui pèsent sur le CAP. Plus encore, en contrôlant ces variables, le chercheur peut étudier l'impact sur ce CAP d'une variation à la marge de l'une de ces variables décisionnelles toutes choses étant égales par ailleurs.

La première partie de cet article présente l’intérêt pour le décideur public d’observer les CAP individuels. Après avoir exposé les limites des méthodes déclaratives, plus traditionnelles, que sont les enquêtes et les évaluations contingentes (deuxième partie), nous montrons l'intérêt de recourir à la méthode expérimentale pour évaluer les préférences individuelles. Cette troisième partie, plus méthodologique, porte sur la mise en œuvre des études expérimentales visant à mesurer des CAP. En particulier, nous traitons des principaux éléments de design pour ce type d'études. Nous conclurons en traitant des limites rencontrées dans la mise en œuvre de cet outil de recherche.

\section{Les consentements à payer individuels intéressent le décideur public}

Les préférences des consommateurs intéressent le décideur public. Tout d'abord, les pouvoirs publics interviennent directement et de multiples manières sur les marchés (les définitions des produits, les modes de vente, les structures de l'offre, l'information disponible, etc.) ou directement sur les prix (contrôle, fiscalité, subventions). L’efficacité de ces interventions passe par une bonne connaissance des comportements des consommateurs et des préférences qui les sous-tendent. Ainsi les marchés finaux sont-ils encadrés par une législation de plus en plus importante de protection du consommateur. Or, certaines notions générales du droit de la concurrence renvoient directement aux comportements des consommateurs et aux perceptions qui fondent ces comportements. Par exemple, il existe une réglementation pour protéger le consommateur contre les allégations trompeuses sur les produits offerts à la vente. 
Pour appliquer judicieusement cette réglementation les pouvoirs publics devraient pouvoir disposer de procédures rigoureuses et scientifiquement reconnues permettant d'observer que les consommateurs sont effectivement trompés. Ainsi, on pourrait souhaiter disposer d'un outil permettant de montrer clairement, le cas échéant, que telle allégation sur un produit a pour seul effet de jeter inutilement le discrédit sur les produits concurrents. Les pouvoirs publics sont également à l'origine ou sont en position d'encadrer de très près certains signes de qualité : produits d’appellations contrôlées, produits issus de l'agriculture biologique, produits du commerce équitable, pour n'en citer que quelques uns. Là encore, une bonne connaissance des comportements des consommateurs face à ces signes conduira les pouvoirs publics à mieux légiférer. Par ailleurs, les interventions publiques les plus classiques sont également concernées. Les outils fiscaux comme les taxes et les subventions sélectives sont des moyens réputés puissants pour peser sur les comportements des consommateurs. L'augmentation du prix des cigarettes l'a montré au cours de ces dernières années. On le voit, les pouvoirs publics, parce qu'ils ont comme mission d'encadrer les marchés finaux, mais aussi parce qu'ils peuvent souhaiter intervenir directement sur ces marchés, ont des raisons qu'on pourrait qualifier de directes de s’intéresser aux préférences individuelles et aux comportements des consommateurs. L'observation et l'analyse de ces comportements directement sur les marchés peuvent poser des problèmes méthodologiques. Du fait du manque de contrôle sur les variables entourant ces comportements, il est difficile de mesurer l'influence d'une variable isolée «toutes choses égales par ailleurs » sur les comportements des individus. Mais plus encore, il est souvent coûteux, voire inenvisageable d'opérer une étude d'impact sur la base d'une expérience de terrain. Pour anticiper les effets d'une nouvelle mesure encadrant la vente de produits, le décideur public doit alors se tourner vers d'autres méthodes.

Une seconde raison, qu'on pourrait qualifier d'indirecte, permet de justifier l'intérêt des pouvoirs publics pour la connaissance des préférences individuelles. Dans les économies de marché contemporaines, les pouvoirs publics sont amenés à encadrer la production et la consommation d'une grande quantité de biens qui sont des biens non marchands (dont certains sont des biens publics). Pour ces biens, il n'existe pas de prix défini par une confrontation entre une offre et une demande qu'il serait possible d'observer directement sur un marché. Pour encadrer correctement la production et la mise à disposition de ces biens et services non marchands, les pouvoirs publics ont ainsi besoin d'évaluer leur valeur pour les futurs usagers. Ainsi par exemple, l'évaluation de la valeur privée qu'engendrerait 
l'amélioration d'un paysage ou la réduction d'une pollution est utile. L’utilité d'une appréciation de cette valeur sera d'autant plus grande qu'une telle amélioration d'un paysage ou d'une réduction d'une pollution se traduit souvent par des effets marchands directs accroissement des coûts de production de biens marchands qui engendrent ces effets indésirables par exemple - et qu'il est alors légitime, pour les pouvoirs publics, de chercher à faire le bilan le plus complet possible des effets d'une intervention sur le bien-être. Mais les pouvoirs publics vont alors être confrontés à une épineuse question méthodologique : comment évaluer la valeur d'un bien non marchand ? En d'autres termes, comment connaître le prix de marché, c'est-à-dire le prix que les consommateurs et les usagers seraient prêts à payer pour un bien ou pour un service, alors même que le bien ou le service concerné ne sera jamais mis sur un marché ?

Pour un économiste, les préférences des individus s'expriment au travers des comportements d'achat sur les marchés. L'analyse des préférences peut ainsi être faite par l'étude des transactions et en particulier au travers du CAP ou du prix limite des individus. Cette approche des préférences est malaisée même lorsque l'on dispose d'un marché de référence, car elle suppose la connaissance des variables qui concourent à la formation des CAP. Or, sur les marchés, ces variables sont nombreuses et souvent non observables. L'analyse des CAP sur la base des comportements d'achat effectifs est ainsi difficile à conduire. Pour certains biens, la mesure directe du CAP est même absolument impossible du fait de l'inexistence de marché, Bonnieux et al. (1995) parlent à leur propos de bien "hors marché". Certains produits en cours de développement n'ont pas encore de marché, d'autres ne sont pas encore autorisés à la vente. Enfin, dans certains cas, les biens ne seront jamais échangés sur un marché. C’est souvent le cas des biens publics. L’absence de marché de référence est donc bien une situation commune à laquelle doit faire face le décideur public. Comment estimer les préférences individuelles pour un bien environnemental, pour un équipement public ou pour un choix sanitaire ? Plus précisément, dans le cadre d'une analyse coût-bénéfice d'une décision publique, comment évaluer le CAP des personnes affectées par cette décision ? Toute la difficulté et l'enjeu consistent alors à obtenir une estimation monétaire la plus proche possible du prix de marché, si ce marché existait. 
La mesure du CAP par questionnaire : les limites des méthodes déclaratives

Lorsqu'il n'est pas possible de déduire les CAP de l'observation directe des comportements d'achat sur les marchés, il est souvent possible de mobiliser d'autres méthodes. L’une d'entre elles, largement utilisée, est la méthode d’évaluation contingente. Elle permet de révéler les préférences exprimées directement par les individus. Elle se fonde sur le relevé des intentions déclarées par les individus et non pas sur des comportements effectifs $^{2}$. Comme d'autres méthodes déclaratives, la méthode d’évaluation contingente est sujette à un certain nombre de biais que nous allons passer en revue maintenant ${ }^{3}$.

Le biais hypothétique. Un premier problème inhérent aux méthodes déclaratives est lié au fait que les individus ne font pas face à un choix réel pouvant avoir une implication directe sur leur bien-être. On parle de biais hypothétique pour désigner la différence entre un choix hypothétique et un choix réel. Ainsi, il existe une différence significative entre un CAP obtenu dans un contexte hypothétique, comme lors d'une enquête, et celui mesuré dans un contexte réel, comme sur un marché. Pour illustrer l’importance de ce biais, nous présentons ci-dessous les observations obtenues dans le cadre d'une étude expérimentale conduite en 2005 dans le laboratoire du BETA à Strasbourg (Robin et Rozan, 2005). Au cours de cette étude, chaque participant devait, successivement, déclarer le montant maximum hypothétique qu'il serait prêt à payer pour acquérir un DVD (CAP hypothétique) puis les mêmes participants devaient faire une offre d'achat dans le cadre de vente réelle organisée selon des règles incitant à révéler son prix limite d'achat (CAP réel). L'existence de cette seconde mesure ne pouvait être anticipée par les participants au moment de la mesure hypothétique. Comme le montre le graphique 1, la différence entre le CAP hypothétique et le CAP réel est significative.

\footnotetext{
${ }^{2}$ Pour une présentation de la méthode, on se reportera au manuel de Desaigues et Point (1993). Pour une illustration de cette démarche, voir Lusk et Rozan (2005).

${ }^{3}$ Pour une présentation complète de ces biais voir Mitchell et Carson (1989) et Bonnieux, et al. (1995).
} 


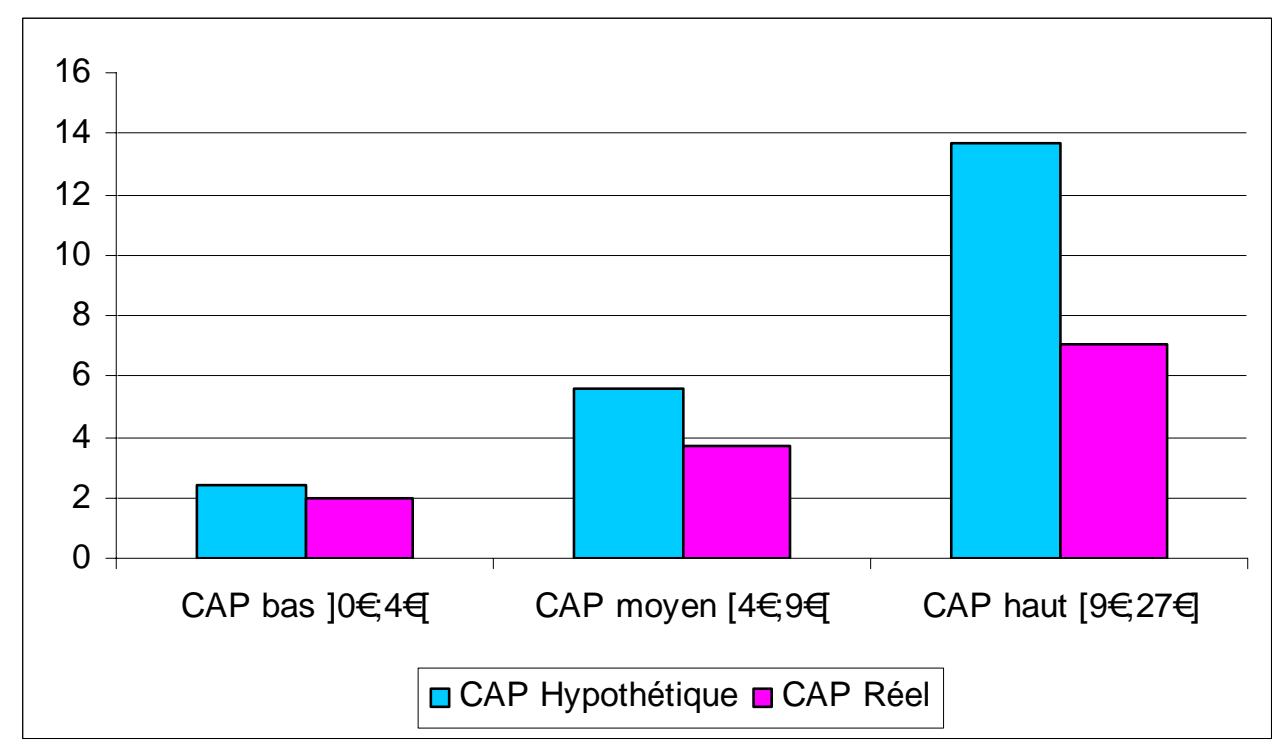

Graphique 1. Comparaison CAP hypothétique et CAP réel (Robin et Rozan, 2005)

Conformément à d'autres études précédemment conduites sur cette question, on observe que la quasi-totalité des participants indiquent un CAP hypothétique supérieur au CAP réel. En moyenne, le biais hypothétique induit une surestimation de $30 \%{ }^{4}$.

Lusk (2003) montre que le biais hypothétique peut être significativement réduit, grâce à l'introduction d'un cheap talk. Dans un questionnaire d'évaluation contingente, l'auteur a introduit un texte expliquant aux répondants l'existence du biais hypothétique et ses conséquences. Il explique qu'il faut répondre à la question de l'évaluation comme si on se trouvait dans un magasin et se demander combien on paierait réellement ce produit. Ces recommandations ont un impact significatif sur la mesure du CAP individuel et peuvent permettre de réduire en partie l'importance du biais hypothétique. Cette réduction n’est néanmoins pas totale et reste bruitée au plan individuel.

L'effet Warm Glow. L'une des critiques les plus fortes concernant les méthodes déclaratives porte sur le fait que les individus révèleraient une opinion ou un jugement qui ne serait pas totalement représentatif de leur préférence réelle. Ce point est particulièrement important quand il s'agit d'estimer le CAP pour un bien public. Ainsi, pour une question relative à une mesure environnementale, un individu interrogé exprimerait son jugement quant à la valeur de la politique de préservation proposée plutôt que la valeur intrinsèque personnelle pour l'actif qui constitue l'objet de cette politique. Le sujet se comporte ainsi, comme le suggère Ruffieux (2004), en « consommateur-citoyen ». Ainsi, dans une étude sur

\footnotetext{
${ }^{4}$ Pour une revue de la littérature sur les études expérimentales du biais hypothétique voir Harrison et Rutström (forthcoming), Lusk et al. (2005).
} 
les préférences individuelles face aux organismes génétiquement modifiés, Noussair et al. (2003), (2004a), observent des différences significatives entre l'opinion des individus, très hostile aux aliments intégrant des organismes génétiquement modifiés, et les comportements d'achat de ces mêmes individus dans des ventes conduites en laboratoire montrant une plus grande acceptation de ces aliments. L'achat d'une bonne conscience (Kahneman et Knetsch (1992)) ou l'argument du don (Diamond et Hausman (1994)) alimentent également la critique. Selon ces auteurs, le CAP ne révèle pas réellement une variation de bien-être individuel mais plutôt une satisfaction liée à la 'bonne action' sous-jacente à l'expression d'une préférence en faveur du bien-être social. A ce titre, les CAP déclarés ne pourraient être utilisés directement comme un indicateur du bénéfice induit par la fourniture d’un bien collectif.

Le biais stratégique. Face à un bien public, si un individu pense que l'accès au bien estimé sera gratuit, le comportement stratégique sera alors de surestimer son CAP. A l'inverse, si le répondant anticipe un accès payant, il aura tendance à sous-estimer son CAP. Le biais stratégique renvoie à ce comportement de passager clandestin consistant à souhaiter profiter d'un bien public sans contribuer à en payer le prix. La question de la contribution volontaire à un bien public a été largement étudiée par l'économie expérimentale (Davis et Holt (1993), Ledyard (1995)). Ces travaux ont montré qu'en laboratoire, si le comportement de passager clandestin n’est pas systématique, il est néanmoins bien présent dans les contributions individuelles à un bien public. En conséquence, une telle logique peut jouer et doit donc être anticipée dans les réponses attendues d'un questionnaire d'évaluation contingente.

Le biais d'ancrage. La question de la révélation des préférences se trouve elle aussi au cœur d'une controverse portant sur le biais d'ancrage. Face à la question ouverte sur son CAP - question à laquelle on l'a vu le consommateur n'est en général confronté que de façon indirecte à travers l'acceptation ou le refus d'un prix affiché - la personne interrogée peut éprouver des difficultés à répondre. La décision la plus courante d'un consommateur consiste à accepter ou à refuser un bien qui lui est offert doté d'un prix, visible sur une étiquette ou sur un catalogue. Pour pallier à cette difficulté, le rapport du NOAA Panel (Arrow et al. (1993)) prône le recours à la question fermée, appelée encore question du référendum puisqu'elle appelle une réponse oui/non à une offre proposée dans le questionnaire (Par exemple, « Etesvous prêt à payer $20 €$ pour le bien ? »). Le problème avec la question fermée est qu'elle donne la position du CAP individuel par rapport à la valeur proposée et non pas la valeur 
précise du CAP. L’information recueillie est donc très pauvre. L’usage des questions fermées itératives s’est alors développé, le CAP étant peu à peu approximé par une série de valeurs proposées successivement au répondant. Cependant, cette démarche pose un problème de biais d'ancrage mis en évidence par Herriges et Shogren (1996). Dans la succession des questions, les individus se réfèrent à la première offre proposée interprétant cette offre comme le montant auquel les ménages sont sensés contribuer ${ }^{5}$. Le CAP finalement estimé est significativement dépendant de cette première offre.

La mesure des CAP dans le cadre d'une évaluation contingente est potentiellement sujette aux différents biais que nous venons de présenter. L’importance de ces biais est variable en fonction des individus ou du contexte de l'étude. En conséquence, la correction de ces biais de mesure est complexe et malaisée. Les problèmes que posent l'interprétation et l'utilisation des CAP obtenus sur la base de méthodes déclaratives ont suscité le développement de nouvelles méthodes de mesure en laboratoire inspirées par les recherches en économie expérimentale.

\section{Mesurer les préférences en laboratoire}

Dans cette partie, nous présentons et discutons de la manière de mesurer les CAP en laboratoire d'économie expérimentale. Les premières études reposant sur la mesure des préférences individuelles en laboratoire pour des biens réels datent des années 70 (Bishop et Heberlein (1979), Bohm (1972)). Ces auteurs utilisaient les avancées alors récentes de l’économie expérimentale des années 60. Le principe général était simple. Plutôt que de confronter les individus à des situations de choix hypothétiques comme c'est le cas en évaluation contingente, il fallait proposer des choix réels ayant des implications directs sur le bien-être des personnes participants aux études. Plutôt que de demander à une personne combien elle accepterait de payer pour obtenir un bien ou un service, il fallait lui proposer d’acheter réellement ce produit et mesurer le prix qu’elle acceptait effectivement de payer.

Le recours à l'économie expérimentale pour évaluer les préférences des consommateurs offre plusieurs atouts.

Tout d'abord, cette méthode permet d’obtenir des estimations réelles, c'est-à-dire de véritables prix issus de transactions effectives par opposition aux CAP hypothétiques obtenus

\footnotetext{
${ }^{5}$ De plus, face à ce type de question fermée, certains individus ont tendance à acquiescer soit pour faire plaisir à l'enquêteur, soit pour obtenir une approbation sociale (ce dernier phénomène est qualifié de " yea saying », par Kanninen (1995)
} 
par évaluation contingente ou par d'autres méthodes déclaratives. Le participant à une étude en laboratoire qui déclare son CAP via une offre sur une enchère par exemple, sait que son niveau de bien-être peut significativement être modifié par sa décision. L’économie expérimentale permet ainsi d'abriter la mesure du CAP du biais hypothétique mais aussi de l'effet de Warm Glow. De même, en liant directement le CAP d'un individu à son niveau de bien-être individuel, l'étude en laboratoire échappe en partie au biais stratégique.

En plus de limiter les biais de mesure, la méthode expérimentale permet d'accroître sensiblement le degré du contrôle de l'environnement dans lequel est effectuée cette mesure. Il est possible, par exemple, de contrôler l'information qui est donnée aux participants et de faire varier cette information durant l'expérience. L'expérience peut aussi être répliquée en maintenant à l'identique les conditions de mesure. Par rapport aux conditions classiques de passation de questionnaire, l'étude en laboratoire permet de concevoir un environnement d'étude contrôlé et répondant aux attentes du chercheur.

Compte tenu du nombre considérable d'études des CAP en laboratoire qui ont été conduites, il n’est pas possible de faire ici une revue exhaustive de la littérature. Nous nous contenterons d'en citer quelques unes afin d'illustrer la grande variété des questions traitées. Une partie de ces études porte sur la mesure des CAP pour des biens publics. Citons à titre d'exemples l'étude de Bohm (1972) portant sur la mesure des CAP pour un nouveau programme TV proposé à des habitants de Stockholm ou celle de Brookshire et Coursey (1987) portant sur la modification d'un parc d'agrément. Dans leur grande majorité, les études en laboratoire ont été consacrées à l'analyse des comportements d'achat de produits alimentaires dont la consommation peut présenter un risque objectif ou subjectif pour la santé. Pour des biens déjà mis en vente sur le marché, l'économie expérimentale permet surtout de focaliser l'observation sur le CAP pour une caractéristique bien précise. Ainsi, pour illustrer la diversité des champs d'investigations, citons les études qui ont portées sur l'acceptabilité pour des aliments provenant d'animaux élevés avec utilisation d'hormones de croissance (Alfnes et Rickertsen (2003), Buhr et al. (1993), Fox et al. (1994)) pour des fruits présentant des traces d'insecticide ou de métaux lourds (Roosen et al. (1998), Rozan et al. (2004)), pour un aliment sur lequel pèse le risque de la présence d’un pathogène (Buzby et al. (1998), Hayes et al. (1995)) ou encore pour des aliments intégrant des organismes génétiquement modifiés (Lusk, et al. (2005), Noussair, et al. (2004a)) ${ }^{6}$.

\footnotetext{
${ }^{6}$ Le lecteur pourra aussi se référer à la revue de littérature coordonnée par Lusk (Lusk et al., 2005).
} 
Nous commencerons cette partie en présentant un exemple de mesure du CAP en laboratoire qui nous permettra d'exposer la logique générale de la méthode. Nous traiterons ensuite des principaux éléments méthodologiques de ce type de démarche avant de terminer par un exposé de ses limites.

\section{Un exemple de mesure du consentement à payer en laboratoire}

Le principe général de la mesure du CAP en laboratoire repose sur les propriétés révélatrices de ce CAP de certaines procédures de vente. L'enchère de Vickrey est de ce point de vue exemplaire. Cette procédure de vente est une enchère sous pli fermé au plus offrant au second meilleur prix. Dans une vente organisée avec cette enchère, un acheteur rationnel fait une proposition d'achat qui correspond à son CAP. Si un acheteur fait une proposition d'achat supérieure à ce montant il risque en effet inutilement d'acheter le bien mis en vente à un prix supérieur à son prix limite d'achat. Symétriquement, s’il fait une proposition d'achat inférieure à son CAP, il risque inutilement de rater une opportunité d'achat à un prix inférieur à son prix limite $^{7}$. En théorie donc, pour des individus aux comportements d'achat rationnels, pour obtenir des estimations sans biais de leurs CAP pour un bien donné, il suffit d'organiser une vente de ce bien en laboratoire en utilisant l'enchère de Vickrey.

$\mathrm{Au}$ moment où il fait une proposition d'achat, le participant à cette vente doit être convaincu que, s'il remporte l'enchère, la vente sera effective. En d'autres termes, en décidant du montant qu'il propose pour acheter le bien, un individu doit savoir que sa décision aura un impact direct sur son bien-être. Si sa proposition est supérieure à toutes les autres offres, il devra acheter : il payera le prix de la seconde meilleure proposition et il recevra effectivement le bien. Pour l'observateur, le CAP de l'individu est estimé directement par sa proposition d'achat. En dehors d'inciter les individus à faire des propositions d'achat sincères et crédibles, la conduite de cette vente en laboratoire permet d'opérer un contrôle strict sur l'environnement de la vente en particulier sur l'information communiquée aux participants en ce qui concerne le bien. Ce contrôle permet de répéter des mesures dans des conditions quasi-constantes autorisant, par exemple, des mesures d'impact sur le CAP d'informations additionnelles sur le bien mis en vente.

Comme nous l'avons indiqué précédemment, si les individus se comportent rationnellement au sens de la théorie, alors la proposition d'achat faite dans une enchère de

\footnotetext{
${ }^{7}$ Pour une démonstration formelle des propriétés de l'enchère de Vickrey, on pourra se référer à l'article de ce dernier Vickrey (1961).
} 
Vickrey donne une mesure directe et non biaisée du CAP. Malheureusement, de nombreuses études en laboratoire ont montré que pour les enchères, comme pour d'autres situations économiques, les individus prennent des décisions qui s'écartent des choix rationnels prédits par la théorie. Se pose alors la question de la pertinence de l'offre d'achat comme estimateur du CAP et du choix de la procédure de vente.

\section{Le choix de la procédure de vente et l'influence de ce choix sur le consentement à payer}

La théorie des enchères nous offre de multiples solutions qui permettent d'estimer les CAP individuels sur la base des offres d'achat. Selon la théorie, pour des individus rationnels, le choix de la procédure de vente est neutre pour ce qui est de la qualité de la mesure du CAP. Mais les résultats obtenus en laboratoire pour des biens à valeurs induites ${ }^{8}$ montrent que les offres d'achat observées s'écartent significativement des CAP réels des individus. Les expériences conduites avec des biens réels montrent que la mesure absolue du CAP est sensible au choix de la procédure de vente. Enfin, il apparaît que l'écart entre l'offre effective et le CAP peut varier selon que le participant à la vente a le sentiment qu'il attribue une valeur faible ou une valeur forte par rapport aux autres participants. Cette influence de la croyance d'un individu quand à la position relative de son CAP est, elle aussi, sensible à la procédure utilisée.

Commençons par l'enchère de Vickrey que nous avons présentée dans notre exemple. Il s’agit sans doute de la procédure de vente la plus couramment utilisée pour les mesures de CAP en laboratoire. Cependant les études conduites avec des biens à valeurs induites montrent que les offres d'achat observées s'écartent significativement des prix limites. Ainsi, tout en reconnaissant les bonnes propriétés globales de l'enchère de Vickrey, Kagel et al. (1987) et Kagel et Levin (1993) observent que les acheteurs qui remportent l'enchère font des offres d'achat significativement supérieures à leur prix limite. Par ailleurs, cette enchère ne permet pas de motiver les acheteurs dont le CAP est faible. En effet, ces derniers, anticipant qu'ils ne pourront jamais acquérir le bien mis en vente, ne sont pas incités à faire une offre correspondant à leur valeur limite d'achat. Ce phénomène est qualifié de throwaway bid (Fox et al. (1992), Harstad (2000)). Pour inciter les participants à faible valeur à faire des offres

\footnotetext{
${ }^{8}$ Pour l'étude des comportements d'achat, on peut distinguer les expériences conduites avec des biens fictifs pour lesquels les sujets ont des valeurs induites (induced value), pour lesquels l'expérimentateur contrôle le prix limite d'achat du sujet, des expériences conduites avec biens réels pour lesquels les sujets ont une valeur qui leur est privée (homegrown value) que l'expérimentateur cherche à connaître. Bien entendu, conduire des expériences avec des valeurs induites est le meilleur moyen pour mesurer l'écart possible entre l'offre d'achat et le prix limite de chaque individu.
} 
d'achat proche de leur CAP effectif, il est possible d'accroître le nombre de participants qui achètent dans une enchère de Vickrey. Ainsi dans une vente à dix participants, il est possible de vendre aux cinq meilleurs offrants au sixième meilleur prix. Horowitz et McConnell (2002) ont montré que si l'on gagne en incitation sur les participants à valeur basse, on perd cependant en incitation pour les acheteurs à valeurs hautes.

Partant de ce constat, le mécanisme dit 'BDM’ proposé par Becker et al. (1964), semble constituer une alternative intéressante. Dans cette procédure, les acheteurs font une offre sous pli fermé. Une fois les offres collectées, un prix de vente effectif est tiré au sort dans un ensemble de prix possibles préalablement défini. Les acheteurs ayant fait une offre de prix supérieure au prix tiré au sort achètent le produit à ce prix, les autres n’achètent pas. En théorie, cette procédure, comme l’enchère de Vickrey, a la propriété de révéler le CAP d'un individu rationnel. Tous les participants, quel que soit le niveau auquel ils valorisent le bien, ont une probabilité non nulle d'acheter le bien à un prix inférieur à leur offre. Irwin et al. (1998) montrent que ce mécanisme peut donner de bons résultats dans certaines distributions de prix. En revanche, Bohm et al. (1997) suggèrent que le mécanisme BDM peut ne pas être efficace pour révéler les CAP si l'ensemble des prix au sein duquel s'effectue le tirage au sort est choisi de façon inappropriée.

La comparaison directe de l'enchère de Vickrey et du mécanisme BDM semble cependant montrer qu'en moyenne, l'enchère de Vickrey donne une meilleure estimation des CAP individuels. Noussair et al. (2004b) proposent une comparaison des deux procédures de vente dans le cadre d'une procédure d'apprentissage avec valeurs induites. Cette expérience est conduite avec des participants non étudiants. Les sujets participent à une succession de période de ventes avec des valeurs induites différentes à chaque période. A la fin de chaque période, les offres et les valeurs induites de tous les participants sont affichées au tableau et les sujets ont l'opportunité de discuter ces résultats. On observe alors que des sujets comprennent rapidement quelle est la stratégie optimale pour l'enchère de Vickrey et qu'après quelques périodes ils font des offres proches de leur prix limite. En revanche, avec la même procédure d'apprentissage et avec le mécanisme BDM, de nombreux sujets continuent à proposer des offres d'achat significativement différentes de leur valeur limite et cela même après de multiples répétitions. Les auteurs proposent une explication qui repose sur le coût de déviation de l'offre rationnelle dans les deux procédures. Toutes choses étant égales par ailleurs, il est potentiellement plus coûteux de s'écarter de l'offre optimale dans l'enchère de Vickrey que dans le mécanisme BDM. Dans le cadre de ventes répétées, ce coût constitue une incitation forte à adopter la stratégie optimale. Une autre différence notable entre l'enchère de 
Vickrey et le mécanisme BDM est la nature des interactions entre les acheteurs. Dans le mécanisme BDM, les acheteurs ne sont pas véritablement en interaction. En revanche, dans une enchère de Vickrey, les acheteurs sont en concurrence pour l'achat du bien. Ce sentiment de concurrence peut lui aussi constituer une incitation pour les individus à faire des offres proches du CAP.

Shogren et al. (2001) proposent une procédure de vente originale qui mélange l'enchère de Vickrey et la dimension aléatoire du mécanisme BDM. Dans cette procédure, l'enchère au $n^{\text {ième }}$ meilleur prix aléatoire (the random nth-price auction), le prix de vente est fixé aléatoirement mais il est endogène à la procédure de vente. Supposons que $N$ acheteurs participent à la vente. Chaque acheteur fait une offre sous pli fermé. Quand toutes les offres sont collectées et classées par ordre de prix décroissant, on tire au sort un nombre $n$ compris entre 2 et $N$. Les acheteurs ayant fait les $n-1$ meilleures offres achètent et ils payent le $n^{\text {ième }}$ meilleur prix. Les résultats obtenus avec des valeurs induites montrent que l'enchère de Vickrey au second meilleur prix donne de meilleurs résultats, en termes d'estimation des CAP, pour les acheteurs ayant des valeurs limites proches du prix de vente. Au contraire, c'est l'enchère au $n^{\text {ième }}$ meilleur prix aléatoire qui est supérieure pour les acheteurs ayant des valeurs limites faibles et donc éloignées du prix de vente.

Il existe une autre procédure ayant en théorie les mêmes propriétés en termes de révélation des valeurs si les sujets sont rationnels : l'enchère japonaise ${ }^{9}$. Cette enchère fonctionne de la manière suivante : à partir d'un prix de retrait, le commissaire-priseur annonce des prix croissants avec une incrémentation régulière. Pour chaque prix annoncé, chaque acheteur doit déclarer s'il souhaite sortir de la vente ou s’il décide de continuer à participer. Le vainqueur de l'enchère est le dernier acheteur à demeurer dans l'enchère. Le prix d'achat que doit payer le vainqueur est celui auquel est sorti l'avant dernier acheteur. En dehors de l'annonce du prix centralisé et de l'obligation faite aux acheteurs de déclarer s’il quitte ou s’il reste dans l'enchère, cette procédure est très proche d'une enchère anglaise classique ${ }^{10}$ et, d'un point de vue théorique si les sujets sont rationnels, elle doit déboucher sur la même issue. Le résultat de l'enchère anglaise est identique au résultat de l'enchère de Vickrey : le participant dont le CAP est le plus élevé et le prix qu'il paie correspond au prix limite du participant dont le CAP est immédiatement inférieur. L’enchère japonaise à cependant une propriété intéressante que n’a pas l'enchère anglaise. Avec cette dernière, le seul CAP observé est celui du participant au deuxième meilleur prix limite. Dans une enchère japonaise, chaque participant doit, en

\footnotetext{
${ }^{9}$ A notre connaissance et à ce jour, cette procédure n'a pas été utilisée dans les études de CAP.

${ }^{10}$ De fait, l'enchère japonaise constitue la forme facilement modélisable de l'enchère anglaise.
} 
théorie, rester dans la vente tant que le prix annoncé est inférieur à son CAP. En observant le prix auquel chaque participant sort de l'enchère, il est possible d'observer tous les CAP individuels. Une autre caractéristique intéressante dans le déroulement de l'enchère japonaise est qu'elle fonctionne selon une logique de prix affiché plus familière aux personnes non habituées aux salles des ventes. Face à un prix annoncé, il doit décider s’il souhaite acheter à ce prix ou à un prix supérieur ou s'il renonce à l'achat. Ce point est important pour les études de valorisation qui impliquent des consommateurs finals, souvent déstabilisés face à une situation inédite : proposer le prix d'un produit à acheter. Robin et Rozan (2005) comparent les offres obtenues avec l'enchère japonaise, l'enchère de Vickrey et le mécanisme BDM dans le cadre de vente de biens avec valeurs induites. Si l'on compare les écarts moyens entre les offres et les valeurs induites, il apparaît que l'enchère japonaise donne des résultats comparables à ceux obtenus avec l'enchère de Vickrey et que l'on obtient, en moyenne, une meilleure estimation du CAP avec ces deux enchères qu'avec le mécanisme BDM. Si l'on s'intéresse à la proportion de participants adoptant la stratégie optimale, l'enchère de Vickrey apparaît comme la procédure la plus performante. Ainsi, après cinq répétitions, $84 \%$ de participants font une proposition qui correspond à leur valeur induite contre moins de 50\% pour l'enchère japonaise et le mécanisme BDM. Comparée à l'enchère de Vickrey, l'enchère japonaise produit des incitations plus fortes pour les participants à faible valeur mais elle n’incite pas les participants à haute valeur à s'approcher de leur prix limite.

La sensibilité de la mesure du CAP à la procédure utilisée est avérée. L’impact de l'institution retenue pour faire révéler le CAP est un biais que Harrison et al. (2004) qualifient de affiliated beliefs about field prices et dont l'existence serait due au fait qu'il ne s'agit plus d'utiliser ces institutions pour faire révéler des valeurs induites mais bien des valeurs personnelles (homegrown values). Ce résultat renvoie à une interrogation plus fondamentale qui porte sur la formation du CAP pour un individu. Si nous quittons le cadre simple de préférences préexistantes à la situation d'achat, il convient d'admettre que la procédure de vente participe à la formation du CAP. La question du choix de la procédure devient alors d'autant plus importante.

Au total, la mesure du CAP via les offres d'achat dans le cadre d'une vente réelle en laboratoire est une mesure biaisée. Il est important de prendre conscience de l'existence de ce biais, de son importance et de l'influence de la procédure de vente sur ce biais. Prévoir une procédure d'apprentissage avant une étude de valorisation permet de réduire fortement 
l'importance du biais Noussair, et al. (2004b). Par ailleurs, comme nous le verrons dans la suite de cet article, une manière de réduire l'impact de ce biais consiste à effectuer des mesures répétées avec la même procédure en axant l'étude sur l'influence d'une information additionnelle sur l'évolution des offres d'achat.

\section{Mesure par substitution : l'écart entre consentement à payer et consentement à recevoir}

Supposons que l'on cherche à évaluer la valeur qu'un individu attribue à une caractéristique désirable associée à un bien courant ${ }^{11}$. Une méthode possible consiste à doter chaque individu d'un bien ne possédant pas cette caractéristique puis d'estimer le montant monétaire qu'il accepterait de dépenser - son CAP - pour obtenir le même bien avec cette caractéristique. Cette estimation s'effectue par des procédures de vente compatible en incitation poussant les individus à déclarer le montant maximal qu'ils acceptent de dépenser pour passer d'un bien à un autre. On peut aussi mesurer cet équivalent monétaire en procédant de façon inverse. On dote l'individu d'un bien possédant la caractéristique et on évalue le montant monétaire qu’il demande à recevoir, appelé son consentement à recevoir (CAR), pour échanger ce bien contre le même bien n'ayant pas la caractéristique étudiée. Si les effets de richesse sont faibles et s'il existe de nombreux substituts aux biens échangés, la théorie du choix rationnel nous indique qu'un individu rationnel devrait être indifférent entre le montant que l'individu accepte de payer pour obtenir la caractéristique et le montant qu'il demande à recevoir pour renoncer à cette caractéristique. De nombreuses études empiriques montrent néanmoins qu'il existe un écart significatif entre ces deux mesures. Le montant qu'un individu accepte de payer pour obtenir la caractéristique est en général inférieur au montant qu'il demande à recevoir pour abandonner cette caractéristique ${ }^{12}$. L'existence de cet écart pose problème. Si les comportements observés sont qualifiés d'irrationnels ou d'inconsistants par rapport à la théorie du choix rationnel comment savoir alors si les valeurs obtenues dans le cadre de ces études sont une bonne approximation des préférences réelles des individus. Il n'est pas étonnant que cette question fasse l'objet d'une importante littérature et qu'elle motive d'âpres débats parmi les économistes.

\footnotetext{
${ }^{11}$ Cette caractéristique peut être la garantie d'une absence de risque associé à la consommation du bien, une caractéristique environnementale comme un label "bio" ou éthique comme le label "produit équitable".

${ }^{12}$ Pour une revue de la littérature sur l'écart entre CAP et CAR, on pourra se reporter à Horowitz et Mc Connell (2002) ou encore Plott et Zeiler (2003).
} 
L'explication la plus communément avancée sur cet écart entre CAP et CAR repose sur l'effet de dotation (Knetsch (1989)). Un individu est moins disposé à se séparer d'un objet qu'il possède qu'il ne désire acquérir un nouvel objet. Le fait que les préférences individuelles puissent dépendre des dotations initiales des agents viole les hypothèses de la théorie des choix rationnels. Il est possible d'interpréter ces résultats dans le cadre de la Prospect theory selon laquelle les individus sont plus sensibles à une perte qu'à un gain même si la perte est de même ampleur que le gain (Knetsch et al. (2001)). Cette explication, et plus encore l'existence même d'un écart entre CAP et CAR, ne fait pas l'objet d'un consensus parmi les expérimentalistes. Ainsi, Plott et Zeiler (2003) indiquent que l'écart entre le CAP et le CAR n'est pas systématiquement observé dans toutes les expériences. Partant de ce constat, ils en déduisent que cet écart ne peut pas être l'expression d'une caractéristique particulière des préférences humaines. L'explication qu'ils proposent renvoie aux procédures expérimentales utilisées pour effectuer les mesures. Dans le cadre de procédures spécifiques et sur la base de mesures répétées, ils observent que l'écart entre le CAP et le CAR tend à se réduire avec la répétition des échanges. Pour expliquer l'impact de la procédure sur cet écart, les auteurs proposent une hypothèse qu'il nous semble intéressante de reprendre ici. Plutôt que de considérer que les procédures expérimentales incitent les individus à révéler des préférences préétablies, on peut penser qu'elles participent à la mise à jour de ces préférences. Ainsi, face à un produit ou un service nouveau, la procédure utilisée va structurer le processus de décision de l'individu qui permettra à ce dernier d'établir ses préférences pour le produit. Ainsi, en proposant des prix d'achat ou de vente de produit en laboratoire, l'individu effectue une recherche interne pour découvrir véritablement ce qu'il souhaite obtenir.

Le débat sur l'écart entre CAP et CAR n'est pas clos. A ce niveau, nous retiendrons que les mesures de préférences obtenues en laboratoire sont très sensibles aux procédures utilisées. Cela est vrai dans les expériences de substitution de bien, cela est aussi vrai dans les expériences de mesure directe de CAP. Le débat entre CAP et CAR ne concerne évidemment pas que la méthode expérimentale, ce problème a été également soulevées pour la méthode d'évaluation contingente. Ainsi, le rapport du NOAA panel (Arrow, et al. (1993)) recommande d'évaluer le CAP plutôt que le CAR. 


\section{Mesures répétées avec information additionnelle : minimiser l'impact des procédures de} vente

Comme nous l'avons vu, le choix de procédure de vente utilisée pour estimer le CAP a une influence sur le montant absolu de cette mesure. On peut facilement accepter que comme pour tout instrument de mesure, nous ayons une erreur de mesure du CAP par les expériences en laboratoire. Dans l'état actuel des recherches, il n'est pas possible de qualifier et de quantifier cette erreur pour les biens à valeur personnelle privée. Si nous faisons l'hypothèse que cette erreur est stable pour chaque individu, il est possible de réduire son impact de la manière suivante. En procédant par répétition et en portant l'analyse non pas sur le montant absolu du CAP mesuré mais sur l'écart des CAP entre deux répétitions observées auprès de chaque individu, on peut espérer réduire l'influence propre de chaque procédure. L’écart entre deux propositions devient ainsi une estimation de l'évolution du CAP et pour de nombreuses problématiques de recherche, c’est cette évolution plus que le montant absolu du CAP qu'il s’agit de connaître.

Pour illustrer cette démarche, nous reprendrons les protocoles expérimentaux utilisées pour l'étude de la valorisation de la présence d'organisme génétiquement modifié (OGM) dans des produits élémentaires réalisées par Noussair, Robin et Ruffieux en 1999 et 2000 (Noussair et al. (2002), Noussair, et al. (2004a)). Dans ces protocoles, un groupe d'individus échantillonnés sur la base de critères socio-économiques sont invités à faire des offres d'achats pour des produits alimentaires ${ }^{13}$. Les procédures de vente utilisées doivent conduire l'acheteur à faire une proposition égale à son CAP. Chaque participant fait plusieurs propositions successives au cours de plusieurs périodes. A la première période, l'acheteur fait une proposition sur la base d'une dégustation du produit dont une certaine quantité est mise en vente. Aux cours des périodes suivantes, chaque acheteur doit faire des propositions pour les mêmes produits, sur la base d'informations additionnelles relatives à ces produits. Le graphique 2 représente l'évolution des offres moyennes pour un paquet de biscuits secs. La vente 1 est conduite après une dégustation à l'aveugle sans information additionnelle sur le produit (le produit est vu par les sujets, mais sans emballage). La vente 2 est conduite après que les participants aient été informés de la présence d'OGM dans la liste des ingrédients principaux de certains des produits. On observe alors une baisse du montant moyen des propositions pour les produits contenant des OGM de 39\%. Au cours de cette vente, 35\% des participants ayant fait une

\footnotetext{
${ }^{13}$ Via les procédures de recrutements, les chercheurs se sont assurés que les personnes participant à l'étude sont des acheteurs réguliers de ces produits et qu'ainsi, a priori, ils accordent une valeur positive aux produits mis en vente.
} 
proposition positive au cours de la vente 1 décident de ne plus acheter le produit et font une offre nulle. La vente suivante est conduite après que les participants aient lu une note d'information sur les OGM, le génie génétique, les risques et les potentialités de cette technologie. On observe alors une augmentation de l'offre moyenne de $5 \%$ significative mais limitée.

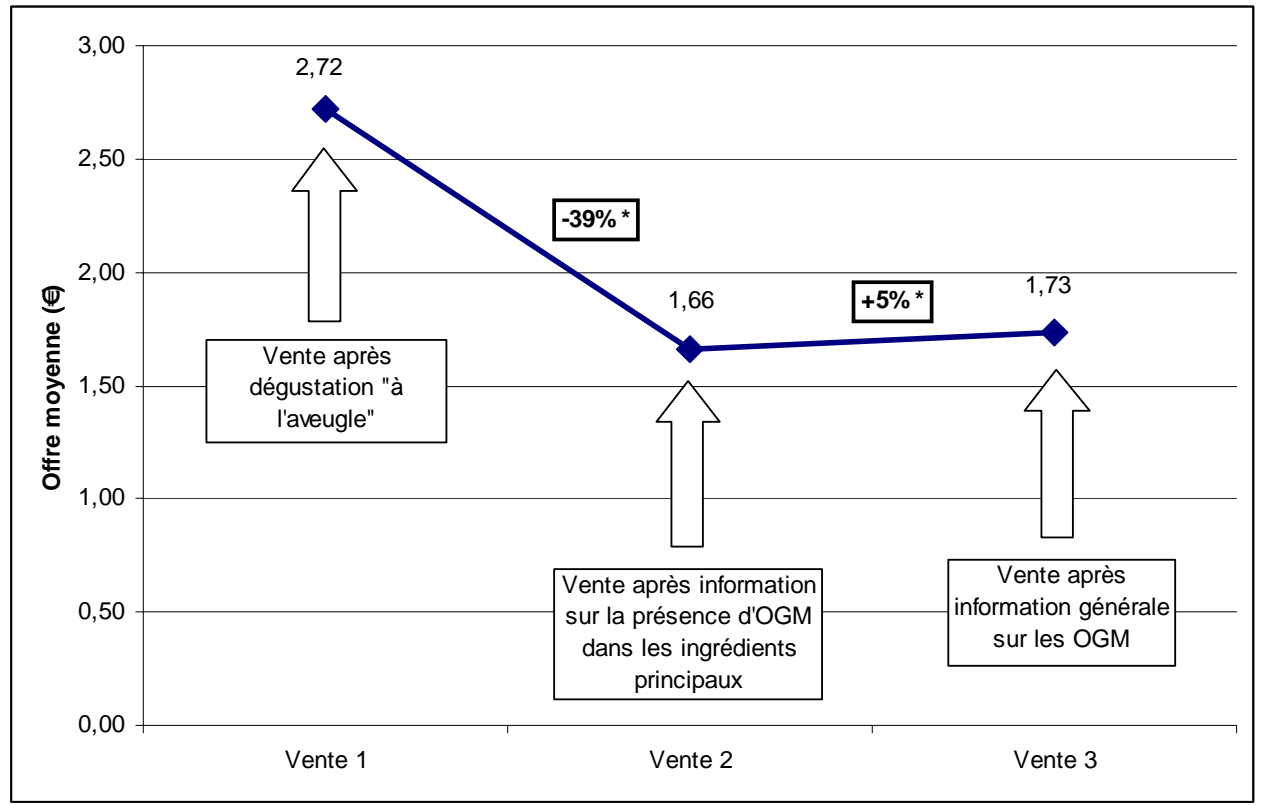

Graphique 2 : Evolution des offres moyennes dans une enchère répétée avec information additionnelle Source : Noussair, et al. (2004a)

Les valeurs encadrées correspondent à l'évolution absolue entre deux ventes, ces évolutions sont statistiquement significatives au seuil de 1\%. L'impact d'une information additionnelle est mesuré en comparant individu par individu, l'offre proposée après information par rapport à l'offre proposée avant information. En dehors de l'information additionnelle, la répétition des ventes est conduite en maintenant un strict contrôle sur les variables qui pourraenit influencer le CAP individuel. Ainsi notamment, les participants sont informés que parmi l'ensemble des périodes de vente, une seule sera tirée au sort à la fin de la session et donnera lieu à une vente effective. De même, pour que le CAP d'un sujet ne soit pas influencé par le CAP des autres sujets, les participants ne reçoivent aucune information sur les offres des autres participants, sur les prix de vente ou sur l'identité des acheteurs effectifs jusqu'à la fin de la session.

Cette démarche par périodes successives permet de réduire l'effet de l'erreur de mesure propre à la procédure de vente utilisée. Ce qui importe ce n'est pas tant l'offre faite à une enchère que l'évolution de cette offre en information croissante. Cependant, ce type de 
protocole contribue à éloigner la situation d'achat en laboratoire de la situation réelle dans laquelle se forment habituellement les CAP.

\section{Effet de contexte et parallélisme : du laboratoire au supermarché}

Le marketing a montré depuis longtemps que le contexte dans lequel se déroule l'achat a une influence significative sur le comportement des consommateurs. De même, de nombreuses études ont établi l'existence d'effet de contexte ou de présentation (framing effect) sur les comportements observés en laboratoire. Qu'en est-il pour les études expérimentales des CAP ? A notre connaissance, il existe peu d'études portant sur cette question. Nous retiendrons l'étude conduite par Lusk et Fox (2003) qui porte sur une importante question: en quoi le CAP d'un consommateur mesuré dans un laboratoire nous informe sur le CAP du même consommateur dans un lieu de vente plus naturel comme un magasin ? Le protocole de l'étude repose sur la conduite de la même expérience dans deux environnements différents. Le premier est un magasin situé sur le campus de l'université d’Etat du Mississippi, le second, plus classique, est celui du laboratoire d'économie expérimentale. Les résultats montrent que le CAP mesuré en magasin est significativement supérieur à celui mesuré en laboratoire. Parmi les explications apportées par les auteurs, nous reprendrons ici celle portant sur l'information sur les prix des substituts d'achat ${ }^{14}$. Cette information est disponible en magasin et non en laboratoire. Elle permet aux sujets de mieux appréhender la valeur du bien vendu sans retarder le moment de l'achat et sans réduire le CAP dans l'attente d'informations supplémentaires. Se pose alors la question du contrôle de la perception de cette information et de son influence sur le CAP.

Si l'on peut facilement admettre que le contexte d'achat influence le CAP d'un individu, rien ne permet de dire quel est le contexte qui permet d'obtenir la meilleure estimation. Là encore, une démarche consistant à mesurer l'impact d'une variable dans le cadre de ventes répétées toutes choses égales par ailleurs constitue sans doute une bonne manière pour réduire l'importance de cet effet de contexte.

\section{Biens privés et biens publics}

Jusqu’à ce jour, les expériences visant à mesurer des CAP individuels, les produits vendus ou échangés étaient dans leur grande majorité des biens privés. Il est plus difficile d'envisager

\footnotetext{
${ }^{14}$ L'autre explication renvoie à un biais de l'étude relative au recrutement des participants. Les participants au traitement en magasin sont des personnes qui sont entrées volontairement dans l'établissement commercial utilisé pour l'étude. De fait ces personnes ont un CAP a priori supérieur aux sujets convoqués pour participer à une étude en laboratoire.
} 
une expérience au cours de laquelle il serait possible d'échanger un bien public. Mis à part quand la caractéristique de non-exclusion du bien public est contournée, permettant la mise en place d'un marché pour le bien (péage d'autoroute par exemple), il n’est par définition pas possible de vendre ce type de bien dans le laboratoire. L’expérimentaliste doit alors faire face à une difficulté assez proche de celle rencontrée par les chercheurs voulant mesurer un CAP pour ce type de bien par la méthode d’évaluation contingente. De fait, les démarches utilisées pour contourner ce problème sont assez similaires. Dans les études expérimentales comme dans les évaluations contingentes, la mesure du CAP pour un bien public s'effectue de manière indirecte par la mesure de CAP pour un bien privé. Plus précisément, la mesure du CAP pour une caractéristique spécifique d'un bien privé permet d'estimer le CAP d'un bien public pour lequel il n'existe pas de marché. Ainsi, pour mesurer le bénéfice d'une amélioration de la qualité de l'air, Chanel et al. (2006), dans leur questionnaire contingent, font révéler le CAP pour quitter un logement situé dans une zone polluée et pour rejoindre un logement dans une zone moins polluée. L’expérimentaliste peut procéder de la même manière. Il peut ainsi par exemple mesurer le CAP d'un individu pour une caractéristique environnementale d'un produit en comparant le CAP pour un produit standard et le CAP pour un produit labellisé bio. Comme pour l'évaluation contingente, deux questions apparaissent. D'une part, comment passer d'une mesure ponctuelle à l'estimation de la valeur globale du bien collectif. D’autre part, quelle est la qualité de la correspondance pour le participant à l'étude entre la caractéristique portée par le produit mis en vente et sa préférence pour le bien public, objet de l'étude.

\section{Conclusion}

Tout au long de cet article, nous avons observé que la méthode expérimentale permettait une mesure des préférences individuelles. Cette méthode constitue non seulement un utile complément voire substitut aux méthodes traditionnelles, mais elle constitue également un puissant stimulant à la réflexion théorique.

La complémentarité aux méthodes traditionnelles est claire : la méthode expérimentale permet non seulement la motivation effective des sujets, mais elle permet aussi de mettre les individus en situation de choix réels. Ces choix se font sur la base d'alternatives concrètes et 
non sur la base de principes généraux comme c’est souvent le cas dans les enquêtes. Un des avantages décisifs de la méthode est que les alternatives proposées aux sujets expérimentés ne sont pas nécessairement des produits ou des services présentés dans leur totalité, mais peuvent être des attributs de produits autorisant une analyse et un affinement potentiellement infini. La méthode se prête ainsi particulièrement bien à la décomposition des produits en caractéristiques. Concernant les caractéristiques environnementales par exemple, celles-ci peuvent être isolées des autres caractéristiques des produits. On peut alors les évaluer en tant que telles, dans un contexte réel et avec des incitations contrôlées.

La méthode expérimentale est aussi un stimulant à la réflexion théorique. Cette réflexion pousse à expliciter certaines limites de la méthode, mais peut-être également certaines limites de la théorie économique elle-même.

Tout d'abord, la méthode a les limites inhérentes à toute mise en maquette d’une réalité plus vaste. Concernant les comportements, la question est de savoir si le protocole adopté est suffisant pour isoler les sujets de variables cachées qui empêcheraient le contrôle correct des comportements. Sait-on, ainsi, si les sujets venus au laboratoire sont motivés par les seuls gains monétaires et les produits proposés à leurs choix, alors que les sommes en jeu et les montants des produits sont généralement faibles? Sait-on dès lors si les sujets ne se comportent pas - comme ils le font pour un questionnaire - dans une position d'expert ou de citoyen et non dans une position de consommateur ${ }^{15}$

Une autre limite due à la mise en maquette est liée à l'écrasement du temps dans le laboratoire. Les sujets expérimentés ont souvent à donner une valeur à des produits nouveaux, à des caractéristiques nouvelles, produits ou caractéristiques auxquelles ils n’ont pas nécessairement réfléchi au préalable, notamment en termes de CAP. Même en admettant que les prix annoncés sont sincères, est-on assuré de la stabilité dans le temps de ces préférences ? Ne seraient-ils pas significativement différents une fois passé le choc initial de la découverte et une fois les routines d'achat installées ? Cette question est évidemment importante pour les caractéristiques des produits qui renvoient à des préférences au contenu collectif (environnementales), futur (innovation) ou incertain (santé).

\footnotetext{
${ }^{15}$ On retrouverait ainsi l'effet de « Warm Glow » qui pèse sur la méthode de l’évaluation contingente.
} 
Une troisième limite de la mise en maquette est l'écrasement des montants en jeu. En effet, de la même manière qu'on écrase le temps, on écrase aussi les budgets dans le laboratoire. Afin de réduire les coûts d'une expérience on tend à formater les expériences sur des petits montants de produits et sur de petites valeurs monétaires. Ceci pose les problèmes d'incitations que nous avons déjà évoqués, mais ceci pose encore un autre problème : celui de la proportionnalité de la valeur des caractéristiques des produits. Prenons par exemple la valeur environnementale d'un produit, sait-on si le CAP d'un sujet pour une caractéristique d'un produit qui épargne l'environnement est bien proportionnelle à la valeur globale du produit ? Supposons qu'une expérience nous montre qu'un sujet est prêt à payer un euro un produit dont le processus de production détériore l'environnement et un euro et vingt centimes pour un produit aux caractéristiques d'usage identiques mais dont le processus de production n’est pas agressif pour l'environnement. Peut-on déduire de ce résultat expérimental que, pour un montant de 1000 euros, ce même sujet sera prêt à dépenser 200 euros pour l'environnement, ou, hypothèse alternative, doit-on en déduire que pour ce montant de 1000 euros du produit expérimental, le sujet ne serait toujours prêt à payer que les 20 centimes observés en laboratoire ?

Ces interrogations sur les limites de l'expérimentation liées en particulier à la qualité des incitations et à la mise en maquette de la réalité que l'on entend indirectement observer de façon contrôlée ne doivent pas dissimuler une question plus importante encore : celle de la nature même de ce qui est révélé par les expériences, du parallèle qu’il y a entre un CAP (variable observée) et les préférences des sujets (variable cachée). Prise au pied de la lettre, la théorie économique standard suggère que la préférence d'un sujet pour une caractéristique quelconque constitue une donnée, donnée qui ne demande qu’à être 'révélée'. Dans un tel cadre théorique, une bonne expérience est une expérience qui révèle correctement cette valeur cachée. A l’inverse, une mauvaise expérience sera qualifiée de 'biaisée’. Mais est-on bien certains que de telles préférences existent ou, plus exactement, préexistent à l'expérience et à son contexte particulier ? Est-on certain qu'elles préexistent en particulier non seulement au produit offert, mais aussi aux conditions institutionnelles particulières de sa mise en marché, bref, à l'expérience ? Sans l'hypothèse de préexistence des valeurs, ce n'est plus de biais dont il faut parler, mais des circonstances particulières dans lesquelles l'expérience a été conduite. Et les résultats ne sont plus généralisables. Il reste à faire de nombreuses expériences, à la fois dans et hors du laboratoire, pour appréhender la stabilité, la sensibilité aux contextes 
institutionnels et informationnels des CAP. Mais l'utilisation future des résultats de laboratoire par les pouvoirs publics de façon fiable est à ce prix.

Bibliographie

Alfnes, F. et Rickertsen, K. (2003) "Willingness to Pay for U.S. Beef in Experimental Auction Markets." American Journal of Agricultural Economics, 85(2), pp. 396-405.

Arrow, K.; Solow, R.; Leamer, E.; Portney, P.; Radner, R. et Schuman, H. (1993), "Report of the Noaa Panel on Contingent Valuation," 64 pages.

Becker, G. M.; DeGroot, M. H. et Marschak, J. (1964) "Measuring Utility by a SingleResponse Sequential Method." Behavioral Science, 9, pp. 226-32.

Bishop, R. C. et Heberlein, T. A. (1979) "Measuring Values of Extramarket Goods: Are Indirect Measures Biased?" American Journal of Agricultural Economics, 61(5, Proceedings Issue), pp. 926-30.

Bohm, P. (1972) "Estimating Demand for Public Goods: An Experiment." European Economic Review, 3(2), pp. 111-30.

Bohm, P.; Linden, J. et Sonnegard, J. (1997) "Eliciting Reservation Prices: BeckerDegroot-Marschak Mechanism Vs. Markets." Economic Journal, 107(443), pp. 1079-89.

Bonnieux, F.; Le Goffe, P. et Vermersch, D. (1995) "La Méthode D'évaluation Contingente : Application À La Qualité Des Eaux Littorales." Economie et Prévision, 117-18(1-2), pp. 89104.

Brookshire, D. S. et Coursey, D. L. (1987) "Measuring the Value of a Public Good: An Empirical Comparison of Elicitation Procedures." The American Economic Review, 77(4), pp. 554-66.

Buhr, B. L.; Hayes, D. J.; Shogren, J. F. et Kliebenstein, J. B. (1993) "Valuing Ambiguity: The Case of Genetically Engineered Growth Enhancers." Journal of Agricultural and Resource Economics, 18(2), pp. 175-84.

Buzby, J. C.; Fox, J. A.; Ready, R. C. et Crutchfield, S. R. (1998) "Measuring Consumer Benefits of Food Safety Risk Reductions." Journal of Agricultural and Applied Economics, 30(1), pp. 69-82.

Chanel, O.; Cleary, S. et Luchini, S. (2006) "Does Public Opinion Influence Willingness-toPay? Evidence from the Field." Applied Economics Letters, 13(13), pp. 821-24.

Davis, D. D. et Holt, C. A. (1993)Experimental Economics. Princeton: Princeton University Press.

Desaigues, B. et Point, P. (1993)Economie Du Patrimoine Naturel: La Valorisation Des Bénéfices De Protection De L'environnement.

Diamond, P. et Hausman, J. (1994) "Contingent Valuation: Is Some Number Better Than No Number ?" Journal of Economic Perspectives, Nov, pp. 681-702.

Fox, J. A.; Hayes, D. J.; Kliebenstein, J. B. et Shogren, J. F. (1994) "Consumer Acceptability of Milk from Coms Treated with Bovine Somatotropin." Journal of Dairy Science, 77(3), pp. 703-07.

Fox, J. C.; Smith, V. L. et Walker, J. M. (1992) "Theory and Misbehaviour of First-Price Auctions: Comment." American Economic Review, 82(5), pp. 1392-412.

Harrison, G. W.; Harstad, R. M. et Rutstrom, E. E. (2004) " Experimental Methods and Elicitation of Values." Experimental Economics, 7(2), pp. 123-40.

Harrison, G. W. et Rutström, E. E. (forthcoming), "Experimental Evidence on the Existence of Hypothetical Bias in Value Elicitation Methods," C. R. P. V. L. Smith, Handbook of Experimental Economics Results. 
Harstad, R. (2000) "Dominant Strategy Adoption and Bidders'experience with Pricing Rules." Experimental Economics, 3, pp. 261-80.

Hayes, D. J.; Shogren, J. F.; Youll Shin, S. et Kliebenstein, J. B. (1995) "Valuing Food Safety in Experimental Auction Markets." American Journal of Agricultural Economics, 77, pp. 40-53.

Herriges, J. A. et Shogren, J. F. (1996) "Starting Point Bias in Dichotomous Choice Valuation with Follow-up Questioning." Journal of Environmental Economics Management, 30, pp. 112-31.

Horowitz, J. K. et McConnell, K. E. (2002) "A Review of Wta/Wtp Studies." Journal of Environmental Economics and Management, 44(3), pp. 426-47.

Irwin, J. R.; McClelland, G. H.; McKee, M.; Schulze, W. D. et Norden, N. E. (1998) "Payoff Dominance Vs. Cognitive Transparency in Decision Making." Economic Inquiry, 36(2), pp. 272-85.

Kagel, J. H.; Harstad, R. M. et Levin, D. (1987) "Information Impact and Allocation Rules in Auctions with Affiliated Private Values: A Laboratory Study." Econometrica, 55(6), pp. 1275-304.

Kagel, J. H. et Levin, D. (1993) "Independent Private Value Auctions: Bidder Behavior in First-, Second-, and Third-Price Auctions with Varying Numbers of Bidders." Economic Journal, 103(419), pp. 868-79.

Kahneman, D. et Knetsch, J. L. (1992) "Valuing Public Goods : The Purchase of Moral Satisfaction." Journal of Environmental Economics and Management, 22, pp. 57-70.

Kanninen, B. J. (1995) "Bias in Discrete Response Contingent Valuation." Journal of Environmental Economics Management, 28, pp. 114-25.

Knetsch, J. L. (1989) "The Endowment Effect and Evidence of Nonreversible Indifference Curves." The American Economic Review, 79(5), pp. 1277-84.

Knetsch, J. L.; Tang, F.-F. et Thaler, R. H. (2001) "The Endowment Effect and Repeated Market Trials: Is the Vickrey Auction Demand Revealing?" Experimental Economics, 4(3), pp. 257-69.

Ledyard, J. O. (1995), "Public Goods: A Survey of Experimental Research," A. Roth etJ. Kagel, Handbook of Experimental Economics. Princeton: Princeton University Press, 111-94.

Lusk, J. L. (2003) "Effect of Cheap Talk on Consumer Willingness-to-Pay for Golden Rice." American Journal of Agricultural Economics, 85, pp. 840-56.

Lusk, J. L. et Fox, J. A. (2003) "Value Elicitation in Retail and Laboratory Environments." Economics Letters, 79, pp. 27-34.

Lusk, J. L.; Jamal, M.; Kurlander, L.; Roucan, M. et Taulman, L. (2005) "A Meta Analysis of Genetically Modified Food Valuation Studies." Journal of Agricultural and Resource Economics, 30, pp. 28-44.

Lusk, J. L. et Rozan, A. (2005) "Consumer Acceptance of Biotechnology and the Role of Second Generation Technologies in the Us and Europe." Trends of Biotechnology, 23(8), pp. 386-87.

Mitchell, R. C. et Carson, R. T. (1989)Using Surveys to Value Public Good: The Contingent Valuation Method. the John Hopkins University, Washington.

Noussair, C.; Robin, S. et Ruffieux, B. (2003) "De L'opinion Publique Aux Comportements Des Consommateurs. Faut-Il Une Filière Sans Ogm ?" Revue Economique, 54(1).

Noussair, C.; Robin, S. et Ruffieux, B. (2002) "Do Consumers Not Care About Biotech Foods or Do They Just Not Read the Labels?" Economics Letters, 75(1), pp. 47-53.

Noussair, C.; Robin, S. et Ruffieux, B. (2004a) "Do Consumers Really Refuse to Buy Genetically Modified Food?" Economic Journal, 114(492), pp. 102-20. 
Noussair, C.; Robin, S. et Ruffieux, B. (2004b) "Revealing Consumers' Willingness-to-Pay: A Comparison of the Bdm Mechanism and the Vickrey Auction." Journal of Economic Psychology, 25(6), pp. 725-41.

Plott, C. R. et Zeiler, K. (2003), "The Willingness to Pay/Willingness to Accept Gap, the "Endowment Effect," Subject Misconceptions and Experimental Procedures for Eliciting Valuations," Caltech Social Science Working Paper. Pasadena, 53.

Robin, S. et Rozan, A. (2005), "Experimental Auction Procedures: A Comparison of the Vickrey Auction, the Japanese Auction and the Bdm Mechanism," Mimeo.

Roosen, J.; Fox, J. A.; Hennessy, D. A. et Schreiber, A. (1998) "Consumers' Valuation of Insecticide Use Restrictions: An Application to Apples." American Journal of Agricultural Economics, 23(2), pp. 367-84.

Rozan, A.; Stenger, A. et Willinger, M. (2004) "Willingness-to-Pay for Food Safety: An Experimental Investigation of Quality Labelling on Bidding Behaviour." European Review of Agricultural Economics, 34(4), pp. 1-16.

Ruffieux, B. (2004) "Le Nouveau Citoyen Consommateur : Que Peut-on En Attendre En Termes D’Efficacité Économique ?" Sciences de la Société, 62(mai), pp. 93-117.

Shogren, J. F.; Margolis, M.; Koo, C. et List, J. A. (2001) "A Random Nth-Price Auction." Journal of Economic Behavior \& Organization, 46(4), pp. 409-21. 